\title{
Erratum to: Highly effective use of Australian Pilbara blend lump ore in a blast furnace
}

\author{
S. Wu, H. Han, X. Liu, H. Wang and F. Xue
}

School of Metallurgical and Ecological Engineering, University of Science and Technology Beijing, Beijing, 100083, China

Revue de Métallurgie 107, 187-193 (2010)

The footnote on page 187 of this article has been inserted by mistake. The editor kindly asks the reader to accept his apologies. 